\title{
Optimal design of hybrid renewable energy system based on solar and biomass for Halal Products Research Institute, UPM
}

\begin{abstract}
This paper presents design optimization of hybrid renewable energy system based on solar and biomass energy resources. The optimization is using HOMER software to get the best and optimal operation system. The optimum sizing of the Hybrid Renewable Energy System (HRES) is determined based on optimization and sensitivity analyses in order to get the best combination or solution of the proposed development. The optimization of HRES considers components selected, its sizing and operational strategy to provide the reliable and efficient system. The excess energy created from the HRES has also been evaluated. It will consider on minimizing the excess energy of the HRES. Data of solar radiation and biomass resources are analyzed and simulated in HOMER to assess the proposed HRES. Overall performance of the HRES will be evaluated and finally analysis will be performed to get the optimal design of the HRES for the pilot area selected.
\end{abstract}

Keyword: Biomass; Excess energy; Hybrid renewable energy system; Optimization; Solar energy 